\section{A suspicious clot}

A 72-year-old man presented with right-sided pleuritic chest pain, chronic cough and weight loss. Chest $x$ ray revealed a moderate sized pleural effusion. Chest CT showed thromboembolism of the right main pulmonary artery, and pleural effusion with compression atelactasis of the right lower lobe of the lung (fig 1). As the thrombus appeared suspicious, being unilateral, central and occupying the entire lumen of the pulmonary artery without abnormality in the contralateral lung, magnetic resonance arteriography was performed which showed heterogeneous enhancement (fig 2). Positron emission tomography (PET)/CT confirmed increased $\left[{ }^{18} \mathrm{~F}\right]$ fluorodeoxyglucose (FDG) uptake by the mass in the right main pulmonary artery, pleura, right clavicle, L1 vertebral body and both adrenal glands (fig 3). He underwent CT-guided biopsy of the L1 vertebral body, and histology was compatible with intimal sarcoma of the pulmonary artery. He was treated with gemcitabine but developed acute dyspnoea with hypotension, and died of heart failure from tumour embolism.

Primary pulmonary artery sarcomas are often misdiagnosed as emboli and suspected when patients do not improve with anticoagulation therapy, which accounts for the advanced stage at diagnosis. ${ }^{1}$ As magnetic resonance imaging, PET and PET/CT are used more frequently in the evaluation of pulmonary

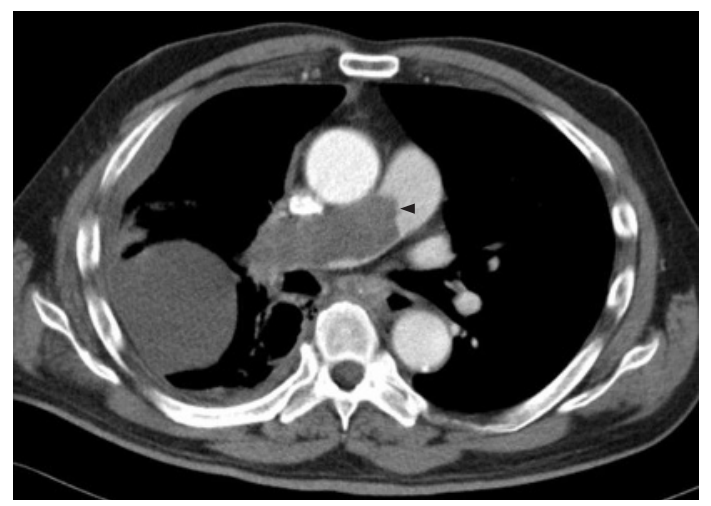

Figure $1 \mathrm{CT}$ at the level of the bronchus intermedius shows a filling defect occupying the entire lumen of the right pulmonary artery (black arrowhead) and right-sided pleural effusion.

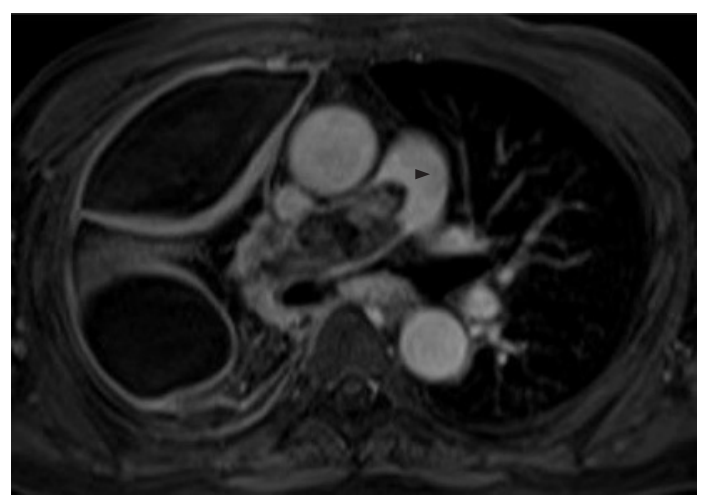

Figure 2 Magnetic resonance arteriography postcontrast-enhanced T1weighted sequence showed heterogeneous enhancement of the intravascular mass (black arrowhead).

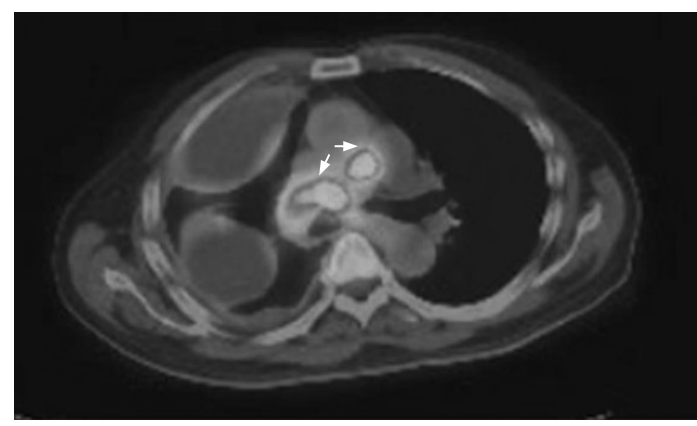

Figure 3 Positron emission tomography/CT showed increased $\left[{ }^{18} \mathrm{~F}\right]$ fluorodeoxyglucose uptake by the mass in the right main pulmonary artery (white arrows).

\section{Learning points}

- Primary pulmonary artery neoplasms are misdiagnosed as pulmonary emboli and suspected when symptoms persist or deteriorate despite adequate anticoagulant or thrombolytic therapy, which accounts for the delay and advanced stage at diagnosis.

- Doctors should be suspicious of pulmonary artery neoplasm if fatigue, weight loss, anaemia and elevated erythrocyte sedimentation rate are detected.

- Enhancement of a filling defect that occupies the entire main or proximal pulmonary artery, extraluminal extension or expansion of the artery on CT alludes to pulmonary artery neoplasm rather than embolus. Pulmonary artery neoplasm is also enhanced on postgadolinium MRI due to its heterogeneous appearance.

- Positron emission tomography (PET), PET/CT may be helpful in differentiating pulmonary artery neoplasm from embolus by demonstrating positive $\left[{ }^{18} \mathrm{~F}\right]$ fluorodeoxyglucose uptake. PET/ CT also facilitates detection of extrathoracic metastasis essential in preoperative staging.

malignancy, it is possible that diagnosis of this rare disease can be made earlier in the clinical course..$^{2-4}$

\section{P Lee, ${ }^{1}$ F-K Cheah, ${ }^{2}$ J Huang, ${ }^{2}$ D Poon, ${ }^{3}$ C-M Loo ${ }^{1}$}

${ }^{1}$ Department of Respiratory and Critical Care Medicine, Singapore General Hospital, Singapore; ${ }^{2}$ Department of Diagnostic Radiology, Singapore General Hospital, Singapore; ${ }^{3}$ Medical Oncology, National Cancer Centre, Singapore

Correspondence to: Dr P Lee, Department of Respiratory and Critical Care Medicine, Singapore General Hospital, Outram Road, Singapore 169608; lee.pyng@sgh.com.sg

Competing interests: None

Patient consent: Obtained.

Accepted 21 January 2009

Thorax 2009;64:1011. doi:10.1136/thx.2008.111211

\section{REFERENCES}

1. Parish JM, Rosenow EC, Swensen SJ, et al. Pulmonary artery sarcoma. Chest 1996;110:1480-8.

2. Yi CA, Lee KS, Choe $\mathrm{YH}$, et al. Computed tomography in pulmonary artery sarcoma: distinguishing features from pulmonary embolic disease. J Comput Assist Tomogr 2004;28:34-9.

3. Kacl GM, Bruder E, Pfammatter T, et al. Primary angiosarcoma of the pulmonary arteries: dynamic contrast-enhanced MRI. J Comput Assist Tomogr 1998;22:687-91.

4. Bruzzi JF, Munden RF. PET/CT imaging of lung cancer. J Thorac Imaging 2006:21:123-36 